\title{
EFEITO DE FIBRAS VEGETAIS NAS PROPRIEDADES DE COMPÓSITOS BIODEGRADÁVEIS DE AMIDO DE MANDIOCA PRODUZIDOS VIA EXTRUSÃO
}

\author{
Effects of vegetal fibers on properties of cassava starch biodegradable \\ composites produced by extrusion
}

\author{
Flávia Debiagi ${ }^{1}$, Suzana Mali², Maria Victória Eiras Grossmann ${ }^{3}$, Fábio Yamashita ${ }^{3}$
}

\begin{abstract}
RESUMO
A preocupação com o volume de lixo tem gerado interesse no desenvolvimento de embalagens biodegradáveis capazes de substituir, ao menos em parte, os plásticos convencionais sintéticos, como é o caso das embalagens de poliestireno expandido (Isopor). Ojvetivou-se,neste trabalho caracterizar, quanto ao índice de expansão (IE), densidade, índice de absorção em água (IAA), índice de solubilidade em água (ISA) e cor (coordenadas $\mathrm{L}^{*}, \mathrm{a}^{*} \mathrm{e} \mathrm{b}^{*}$ ), compósitos biodegradáveis expandidos produzidos via extrusão, a partir da mistura de amido de mandioca, glicerol (plastificante) e dois tipos de fibras vegetais. Os compósitos foram preparados em extrusora mono-rosca, com três diferentes teores de fibras de aveia ou de cana-de-açúcar $(0,5$ e $10 \mathrm{~g} / 100 \mathrm{~g}$ amido), dois teores de umidade (18 e $26 \%$ ) e teor fixo de glicerol $(20 \mathrm{~g} / 100 \mathrm{~g}$ sólidos). A adição das fibras não afetou significativamente o IE, a densidade e o IAA, porém diminuiu o ISA dos materiais, o que é uma vantagem, favorecendo a utilização das fibras no reforço dos compósitos. A adição de fibras levou ao escurecimento das amostras, com decréscimo da luminosidade ( $\left.\mathrm{L}^{*}\right)$ e, ainda, ao aumento nos valores dos parâmetros de cor $\mathrm{a}^{*} \mathrm{e} \mathrm{b}^{*}$. Este estudo é passo fundamental para produção em escala industrial dos compósitos, que necessitam de condições de processo que forneçam resultados reprodutíveis de expansão e capacidade de absorção e solubilidade em água, propriedades de grande importância nesses produtos.
\end{abstract}

Termos para indexação: Embalagens de alimentos, fibras da cana-de-açúcar, fibras da casca de aveia.

\begin{abstract}
The concern with the volume of waste has generated interest in the development of biodegradable packaging able to replace, at least in part, the conventional synthetic plastics, such as packs of expanded polystyrene (Isopor). This study aimed to characterize the expansion index, density, the water absorption index (WAI), water solubility index (WSI) and color (coordinates L*, $\mathrm{a}^{*}$ and $\mathrm{b}^{*}$ ) of expanded biodegradable composites produced by extrusion, from the mixture cassava starch, glycerol (plasticizer) and two different types of vegetable fibres. The composites were prepared in a single screw extruder, with three different levels of oat or sugar cane fibres $(0,5$ and $10 \mathrm{~g} / 100 \mathrm{~g}$ starch), two levels of humidity (18 and $26 \%)$ and a fixed level of glycerol $(20 \mathrm{~g} / 100 \mathrm{~g}$ solds). The addition of fibres did not affect significantly the expansion index, density and WAI of materials, and reduced WSI of products, which is an advantage and promotes the use of these fibres in the reinforcement of composites. The addition of fibers led to the darkening of the samples, with decrease in lightness $\left(\mathrm{L}^{*}\right)$ and increase in the values of the color parameters $\mathrm{a}^{*}$ and $\mathrm{b}^{*}$. This study is a key step for a future industrial scale production of these composites, which requires process conditions that provide reproducible results of expansion and absorption capacity and solubility in water, properties of great importance in these products.
\end{abstract}

Index terms: Food packaging, sugar cane fibers, oat hull fibers.

(Recebido em 1 de abril de 2009 e aprovado em 23 de abril de 2010)

\section{INTRODUÇÃO}

Atualmente, no Brasil, $16,5 \%$ do total de plásticos rígidos e filmes flexíveis produzidos são reciclados, o que equivale a 200 mil toneladas por ano. Contudo, a maior limitação para a reciclagem é a diversidade de polímeros empregados, além da impossibilidade de se usar embalagens recicladas para alimentos, confeccionadas basicamente a partir de polímeros sintéticos convencionais (Mali et al., 2010).

As embalagens plásticas convencionais são formadas por macromoléculas derivadas do petróleo, uma fonte não renovável. Os principais polímeros sintéticos são polietileno (PE), polipropileno (PP), poliestireno (PS), poli (tereftalato de etileno) (PET) e poli (cloreto de vinila) (PVC) (Zhang et al., 2005).

O poliestireno expandido (Isopor), além da dificuldade de reciclagem, por ser de baixa densidade, apresenta um elevado volume, ocupando um espaço maior na natureza quando depositado em lixões ou até mesmo no meio ambiente (Mali et al., 2010). Em busca de soluções para esta questão ambiental, a utilização de polímeros biodegradáveis é uma ótima alternativa para substituir, ao

\footnotetext{
${ }^{1}$ Universidade Estadual de Londrina/UEL - Londrina, PR

2Universidade Estadual de Londrina/UEL - Departamento de Bioquímica e Biotecnologia/CCE - Cx. P. 6001 - 86051-990 - Londrina, PR - smali@uel.br 3Universidade Estadual de Londrina/UEL - Departamento de Ciência e Tecnologia de Alimentos/CCA - Londrina, PR
} 
menos em parte, os polímeros sintéticos convencionais das embalagens plásticas.

Os polímeros biodegradáveis são definidos como plásticos cuja degradação é feita por micro-organismos, como bactérias, fungos ou algas. Compostos de amido, polivinil álcool, polilactatos e polihidroxibutirato podem ser citados como exemplos de polímeros biodegradáveis (Lima, 2004; Bardi \& Rosa, 2007).

O amido é de grande interesse nos estudos dos polímeros biodegradáveis. Apresenta-se abundantemente na natureza e tem baixo custo, podendo, portanto, ser utilizado em escala industrial, além de ser de fonte renovável (Mali et al., 2004, 2005, 2006; Alves et al., 2007; Shimazu et al., 2007; Silva et al., 2007). Entre suas várias aplicações. A de maior interesse seria como matéria-prima para embalagens de fast-food, as quais são muito utilizadas atualmente. Tais embalagens necessitam de baixa resistência mecânica, podendo ser utilizados elevados teores de amido.

Apesar da fácil degradação natural, as embalagens à base de amido apresentam algumas desvantagens. São caras quando comparadas com as embalagens convencionais, não apresentam uma boa flexibilidade e são pouco resistentes à umidade. Para isso, necessitam de tratamentos especiais, como a adição de fibras, plastificantes e outros aditivos, para assim se tornarem resistentes à tração, flexibilidade e ao contato com a água (Salgado et al., 2008).

As fibras da cana-de-açúcar e da casca de aveia são boas alternativas para o tratamento do amido tornandoo tão resistente quanto as embalagens convencionais. Ambas as fibras constam de resíduos industriais abundantes no Brasil, do etanol e da indústria alimentícia, respectivamente (Shibata et al., 2005; Silva et al., 2007).

Objetivou-se, no presente trabalho, caracterizar, quanto à densidade, expansão e capacidade de absorção e à solubilidade em água, compósitos biodegradáveis expandidos produzidos via extrusão, a partir da mistura de amido de mandioca, glicerol (plastificante) e dois diferentes tipos de fibras vegetais, as fibras da cana-de-açúcar e as fibras da aveia.

\section{MATERIAIS E MÉTODOS}

\section{MATERIAIS}

$\mathrm{O}$ amido de mandioca foi adquirido da Indemil Ltda, proveniente de um mesmo lote de fabricação. O bagaço de cana-de-açúcar foi obtido de destilarias próximas à região de Londrina - PR e antes de ser usado foi seco $\left(60^{\circ} \mathrm{C}\right) \mathrm{em}$ estufa de circulação de ar e depois moído. As fibras de casca de aveia foram obtidas de indústrias da região, foram devidamente secas e moídas antes do uso. Todos os reagentes empregados foram de grau analítico.

\section{MÉTODOS}

\section{Produção dos compósitos expandidos}

Os compósitos foram obtidos por extrusão a partir das misturas de amido, fibras e do agente plastificante (glicerol). Foram obtidas amostras controle, sem a incorporação das fibras, para testar o seu efeito e todas as formulações testadas estão contidas na Tabela 1. Foi utilizada uma extrusora de laboratório, marca BGM modelo EL-25 composta por uma rosca única com diâmetro de 250 mm; caixa de alimentação com refrigeração a água; 4 zonas de aquecimento. Foi empregada temperatura de $120^{\circ} \mathrm{C}$, em todas as zonas da extrusora e velocidade do parafuso de $75 \mathrm{rpm}$. A matriz empregada foi uma matriz cilíndrica com 2,8 mm de diâmetro, foram obtidos pellets cilíndricos dos compósitos expandidos.

\section{Caracterização dos compósitos expandidos}

Os compósitos expandidos foram obtidos na forma de pellets cilíndricos, que foram então condicionados em dessecadores, sob umidade relativa de $64 \%$ através do emprego de solução saturada de nitrito de sódio $\left(\mathrm{NaNO}_{2}\right)$ e sob temperatura de $25^{\circ} \mathrm{C}$, em estufa incubadora tipo BOD (Tecnal - São Paulo - Brasil) antes das análises.

\section{Índice de expansão (IE)}

O índice de expansão (IE) foi calculado por meio da relação entre o diâmetro do material extrusado $(\mathrm{mm})$ e o diâmetro da matriz do extrusor ( $\mathrm{mm}$ ). $\mathrm{O}$ valor considerado foi obtido por meio da media aritmética de 20 determinações para cada tratamento, conforme a metodologia proposta por Gujska \& Khan (1991).

\section{Densidade dos compósitos}

A densidade dos materiais foi determinada pela relação entre massa e volume dos compósitos, de acordo com Shogren et al. (1998).

\section{Índice de Absorção em água (IAA) e índice de solubilidade em água (ISA)}

O índice de absorção em água (IAA) e o índice de solubilidade em água (ISA) foram determinados segundo a metodologia de Anderson et al. (1969) com algumas modificações. Em um tubo de centrífuga, previamente tarado, foram colocados aproximadamente $2,5 \mathrm{~g}$ de amostra dos produtos expandidos e $30 \mathrm{~mL}$ de água. Os tubos foram 
agitados por 30 minutos em agitador mecânico e, em seguida, centrifugados a $3000 \mathrm{rpm}$ por 10 minutos. Do líquido sobrenadante, foram transferidos $10 \mathrm{~mL}$, cuidadosamente, para cadinho de porcelana previamente tarado e levado para estufa a $105^{\circ} \mathrm{C}$. O material remanescente foi pesado e o IAA (g água/g sólidos) calculado como segue: $\mathrm{IAA}=(\mathrm{PRC}-\mathrm{PA}) /(\mathrm{PA}-\mathrm{PRE})$, onde $\mathrm{PRC}=$ peso do resíduo de centrifugação $(\mathrm{g}) ; \mathrm{PA}=$ peso da amostra $(\mathrm{g})$ (base seca); e PRE = peso do resíduo de evaporação (g). O Índice de Solubilidade em Água (ISA) foi calculado pela relação entre o peso do resíduo da evaporação e o peso seco da amostra, como segue: $\mathrm{ISA}=(\mathrm{PRE} / \mathrm{PA}) \times 100$, em que $\mathrm{PA}=$ peso da amostra (g) (base seca); e PRE = peso do resíduo de evaporação $(\mathrm{g})$

\section{Cor}

As medidas foram realizadas em colorímetro BYK Gardner (Columbia, EUA), operando no sistema CIE (L*, $a^{*}, b^{*}$ ). Os valores de $L^{*}$ (luminosidade ou brilho) variaram do preto (0) ao branco (100), os valores do croma $a^{*}$ do verde (-60) ao vermelho $(+60)$ e os valores do croma $b^{*}$ do azul ao amarelo, ou seja, de -60 a +60 , respectivamente.

\section{Análise Estatística}

Para análise estatística dos resultados obtidos foi empregado programa computacional Statistica versão 6.0 (Statsoft - Oklahoma - USA). Foi empregado teste de Tukey (nível de significância de 5\%), para comparação de médias.

\section{RESULTADOS E DISCUSSÃO}

Tanto a densidade, quanto o índice de expansão, são propriedades importantes na caracterização de materiais expandidos e, o que se espera, é que as amostras obtidas tenham baixa densidade e elevada expansão, assim como as embalagens de poliestireno expandido (Isopor). $\mathrm{Na}$ extrusão, processo empregado neste trabalho, o material é aquecido em condições adiabáticas e submetido a tensões de cisalhamento que, com a combinação de diferentes tipos de matérias-primas, umidade, temperatura, além de outros fatores intrínsecos da extrusora, propiciam a obtenção de um produto final com características específicas de textura, densidade e expansão. A expansão do material ocorre na saída da extrusora devido à diferença de temperatura e pressão interna e externa, e com o superaquecimento do produto ocorre uma rápida evaporação da água na saída da matriz e a formação de células de ar (Gutkoski \& ElDash, 1999; Lustosa et al., 2008).

Na Tabela 2 estão contidos os resultados de índice de expansão e densidade das amostras. Observou-se que a densidade das amostras variou entre 0,86 a $0,97 \mathrm{~g} / \mathrm{cm}^{3}$, tais valores são similares e até menores do que os encontrados por Schmidt \& Laurindo (2010), que estudou materiais à base de amido para a produção de embalagens expandidas, obtendo valores de densidade entre 0,63 e $1,3 \mathrm{~g} / \mathrm{cm}^{3}$, porém maiores que outros, que obtiveram materiais com densidades entre 0,07 a $0,41 \mathrm{~g} / \mathrm{cm}^{3}$ (Carr et al., 2006; Cinelli et al., 2006). Quando comparados ao poliestireno expandido, que apresenta densidade entre 0,06 e $0,18 \mathrm{~g} / \mathrm{cm}^{3}$ (Glenn et al., 2001), os compósitos de amido foram mais densos, indicando que mais pesquisas devem ser realizadas no sentido de melhorar essa propriedade. A densidade das espumas está diretamente relacionada à viscosidade dos materiais empregados e, de acordo com Shogren et al. (1998), para que o processo

Tabela 1 - Formulação dos compósitos produzidos via extrusão.

\begin{tabular}{cccc}
\hline \multirow{2}{*}{ Amostra } & \multicolumn{3}{c}{ Formulação } \\
\cline { 2 - 4 } & $\begin{array}{c}\text { Fibras } \\
(\mathrm{g} / 100 \text { amido) }\end{array}$ & $\begin{array}{c}\text { Tipo } \\
\text { de fibra }\end{array}$ & $\begin{array}{c}\text { Umidade } \\
\text { (g água /100 g sólidos) }\end{array}$ \\
\hline Controle 1 & - & - & 18 \\
Controle 2 & - & Cana-de-açúcar & 26 \\
Amostra 1 & 5 & Cana-de-açúcar & 18 \\
Amostra 2 & 5 & Casca de aveia & 26 \\
Amostra 3 & 5 & Casca de aveia & 18 \\
Amostra 4 & 5 & Cana-de-açúcar & 26 \\
Amostra 5 & 10 & Cana-de-açúcar & 18 \\
Amostra 6 & 10 & Casca de aveia & 26 \\
Amostra 7 & 10 & Casca de aveia & 18 \\
Amostra 8 & 10 & & 26 \\
\hline
\end{tabular}

Ciênc. agrotec., Lavras, v. 34, n. 6, p. 1522-1529, nov./dez., 2010 
de expansão ocorra de forma adequada, a massa de amido deve ter viscosidade suficiente para reter as olhas de ar formadas durante o processamento, sem colapsar, gerando produtos de baixa densidade. O colapso da estrutura expandida se reflete no aumento da densidade desses materiais.

O IE variou de 2,50 a 2,88, não apresentando variações mediante os tipos diferentes de fibras de teores de umidade (Tabela 2). Portanto, a adição das fibras e a umidade, nas condições empregadas neste trabalho, não afetaram significativamente $(\mathrm{p} \leq 0,05)$ as propriedades de expansão dos materiais obtidos. Nos estudos de Camargo et al. (2008), as propriedades de expansão de amido de mandioca fermentado, adicionado de fibras não foram afetadas pela umidade e pela adição de fibras, assim como neste trabalho. Alguns autores relatam a dificuldade de expansão materiais ricos em fibras (Karam et al., 2001), o que não ocorreu neste trabalho, o que pode estar relacionado aos baixos teores de fibras empregados, no máximo $10 \%$ do total da formulação.

O IAA obteve uma variação de 0,85 a 1,50 g água/ g amostra (Tabela 3). O teor e os tipos de fibras e os diferentes níveis de umidade, nas condições estudadas, não afetaram significativamente $(\mathrm{p} \leq 0,05)$ o índice de absorção em água, como seria o esperado. Segundo Faubion \& Hoseney (1982), o IAA representa um indicativo da disponibilidade das moléculas de grupamentos hidrofílicos (como as hidroxilas), para interagirem com moléculas de água e, com introdução de fibras, o IAA poderia ter diminuído. $\mathrm{O}$ que pode ter ocorrido neste trabalho é que os teores de fibras empregados (5 e 10\%) não foram suficientes para levar a um decréscimo dessa resposta.

O ISA obteve uma variação de 1,44 a $10,30 \%$. Nos compósitos a que não foram adicionados fibras, o índice de absorção de água apresentou-se significativamente maior, portanto, a adição da fibra (independente do tipo) diminuiu o nível de solubilidade dos compósitos. Lue et al. (1991), relataram que ao adicionar fibras pode diminuir a capacidade de interação da água com os materiais amiláceos. Camargo et al. (2008) também observaram um efeito negativo da adição de fibras sobre o ISA das amostras estudadas. No caso de embalagens à base de amido, o decréscimo da solubilidade em água pode ser um indicativo bastante interessante da maior estabilidade desses materiais às diferentes condições ambientais, o que se busca através da incorporação das fibras.

O componente $\mathrm{L}^{*}$, que caracteriza a luminosidade das amostras, variou de 55,45 a 37,50 (Tabela 4). Os maiores valores para luminosidade foram obtidos para as amostras sem adição de fibras, enquanto os menores para as amostras com os maiores teores de fibras, tanto de cana-de-açúcar, quanto de casca de aveia. O tipo de fibra e a umidade não afetaram significativamente $(\mathrm{p} \leq 0,05)$ a luminosidade das amostras. Famá et al. (2009), também observou que a adição de fibras leva ao escurecimento de materiais extrusados, com o decréscimo da luminosidade.

Tabela 2 - Resultados de índice de expansão (IE) e de densidade dos compósitos à base de amido de mandioca e fibras.

\begin{tabular}{cccccc}
\hline \multirow{2}{*}{ Amostra } & \multicolumn{2}{c}{ Formulação $^{\mathrm{a}}$} & \multicolumn{2}{c}{ Resultados $^{\mathrm{b}}$} \\
\cline { 2 - 6 } & $\begin{array}{c}\text { Fibras } \\
(\mathrm{g} / 100 \mathrm{amido})\end{array}$ & $\begin{array}{c}\text { Umidade } \\
(\mathrm{g} / 100 \mathrm{~g} \text { sólidos })\end{array}$ & $\begin{array}{c}\text { Tipo } \\
\text { de fibra }^{\mathrm{c}}\end{array}$ & $\begin{array}{c}\text { Índice de } \\
\text { expansão }\end{array}$ & $\begin{array}{c}\text { Densidade } \\
\left(\mathrm{g} / \mathrm{cm}^{3}\right)\end{array}$ \\
\hline Controle 1 & - & 18 & - & $2,86 \pm 0,30 \mathrm{a}$ & $0,94 \pm 0,06 \mathrm{a}$ \\
Controle 2 & - & 26 & - & $2,55 \pm 0,24 \mathrm{a}$ & $0,89 \pm 0,09 \mathrm{a}$ \\
Amostra 1 & 5 & 18 & CAc & $2,88 \pm 0,30 \mathrm{a}$ & $0,91 \pm 0,08 \mathrm{a}$ \\
Amostra 2 & 5 & 26 & CAc & $2,66 \pm 0,26 \mathrm{a}$ & $0,89 \pm 0,04 \mathrm{a}$ \\
Amostra 3 & 5 & 18 & CAv & $2,80 \pm 0,29 \mathrm{a}$ & $0,97 \pm 0,12 \mathrm{a}$ \\
Amostra 4 & 5 & 26 & CAv & $2,77 \pm 0,33 \mathrm{a}$ & $0,86 \pm 0,04 \mathrm{a}$ \\
Amostra 5 & 10 & 18 & CAc & $2,67 \pm 0,53 \mathrm{a}$ & $0,89 \pm 0,04 \mathrm{a}$ \\
Amostra 6 & 10 & 26 & CAc & $2,55 \pm 0,33 \mathrm{a}$ & $0,89 \pm 0,09 \mathrm{a}$ \\
Amostra 7 & 10 & 18 & CAv & $2,67 \pm 0,36 \mathrm{a}$ & $0,91 \pm 0,01 \mathrm{a}$ \\
Amostra 8 & 10 & 26 & CAv & $2,50 \pm 0,37 \mathrm{a}$ & $0,88 \pm 0,06 \mathrm{a}$ \\
\hline
\end{tabular}

a - Teor de glicerol fixado em $20 \mathrm{~g} / 100 \mathrm{~g}$ de sólidos.

b - Letras minúsculas diferentes, na mesma coluna, indicam diferença significativa entre as amostras (Teste de Tukey, p $\leq 0,05$ ).

c - CAc $=$ cana-de-açúcar e CAv $=$ casca de aveia. 
Tabela 3 - Resultados de índice de absorção de água (IAA) e índice de solubilidade em água (ISA) dos compósitos à base de amido de mandioca e fibras.

\begin{tabular}{cccccc}
\hline \multirow{2}{*}{ Amostra } & \multicolumn{3}{c}{ Formulação $^{\mathrm{a}}$} & \multicolumn{2}{c}{ Resultados $^{\mathrm{b}}$} \\
\cline { 2 - 6 } & $\begin{array}{c}\text { Fibras } \\
\text { (g/100 amido) }\end{array}$ & $\begin{array}{c}\text { Umidade } \\
(\mathrm{g} / 100 \mathrm{~g} \text { sólidos })\end{array}$ & $\begin{array}{c}\text { Tipo } \\
\text { de fibra }^{\mathrm{c}}\end{array}$ & $\begin{array}{c}\text { IAA } \\
\text { (g água/g sólidos) }\end{array}$ & $\begin{array}{c}\text { ISA } \\
(\%)\end{array}$ \\
\hline Controle 1 & - & 18 & - & $0,85 \pm 0,33 \mathrm{a}$ & $4,30 \pm 0,16 \mathrm{~b}$ \\
Controle 2 & - & 26 & - & $1,00 \pm 0,14 \mathrm{a}$ & $10,30 \pm 1,16 \mathrm{a}$ \\
Amostra 1 & 5 & 18 & CAc & $1,14 \pm 0,23 \mathrm{a}$ & $2,23 \pm 0,19 \mathrm{c}$ \\
Amostra 2 & 5 & 26 & CAc & $1,10 \pm 0,25 \mathrm{a}$ & $4,66 \pm 1,16 \mathrm{~b}$ \\
Amostra 3 & 5 & 18 & CAv & $1,17 \pm 0,35 \mathrm{a}$ & $2,30 \pm 0,14 \mathrm{c}$ \\
Amostra 4 & 5 & 26 & CAv & $1,23 \pm 0,29 \mathrm{a}$ & $3,25 \pm 0,84 \mathrm{~b}, \mathrm{c}$ \\
Amostra 5 & 10 & 18 & CAc & $1,50 \pm 0,45 \mathrm{a}$ & $2,25 \pm 0,29 \mathrm{c}$ \\
Amostra 6 & 10 & 26 & CAc & $1,42 \pm 0,41 \mathrm{a}$ & $1,44 \pm 0,28 \mathrm{c}$ \\
Amostra 7 & 10 & 18 & CAv & $1,10 \pm 0,25 \mathrm{a}$ & $2,25 \pm 0,73 \mathrm{c}$ \\
Amostra 8 & 10 & 26 & CAv & $1,50 \pm 0,40 \mathrm{a}$ & $1,58 \pm 0,55 \mathrm{c}$ \\
\hline
\end{tabular}

a - Teor de glicerol fixado em $20 \mathrm{~g} / 100 \mathrm{~g}$ de sólidos.

b - Letras minúsculas diferentes, na mesma coluna, indicam diferença significativa entre as amostras (Teste de Tukey, $\mathrm{p} \leq 0,05$ ).

c - CAc $=$ cana-de-açúcar e CAv = casca de aveia.

Tabela 4 - Resultados dos parâmetros de cor dos compósitos à base de amido de mandioca e fibras.

\begin{tabular}{ccccccc}
\hline Amostra & \multicolumn{3}{c}{ Formulação $^{\mathrm{a}}$} & \multicolumn{3}{c}{ Resultados $^{\mathrm{b}}$} \\
\cline { 2 - 7 } & $\begin{array}{c}\text { Fibras } \\
(\mathrm{g} / 100 \mathrm{amido})\end{array}$ & $\begin{array}{c}\text { Umidade } \\
(\mathrm{g} / 100 \mathrm{~g} \text { sólidos })\end{array}$ & $\begin{array}{c}\text { Tipo } \\
\text { de fibra }\end{array}$ & $\mathrm{L}^{*}$ & $\mathrm{a}^{*}$ & $\mathrm{~b}^{*}$ \\
\hline Controle 1 & - & 18 & - & $55,45 \pm 3,31 \mathrm{a}$ & $1,60 \pm 0,21 \mathrm{~d}$ & $8,70 \pm 0,10 \mathrm{c}$ \\
Controle 2 & - & 26 & - & $52,31 \pm 2,11 \mathrm{a}$ & $2,00 \pm 0,42 \mathrm{~d}$ & $7,25 \pm 0,68 \mathrm{c}$ \\
Amostra 1 & 5 & 18 & CAc & $47,42 \pm 2,33 \mathrm{~b}$ & $8,11 \pm 0,55 \mathrm{c}$ & $18,70 \pm 2,12 \mathrm{~b}$ \\
Amostra 2 & 5 & 26 & CAc & $48,32 \pm 2,05 \mathrm{~b}$ & $8,17 \pm 1,41 \mathrm{~b}, \mathrm{c}$ & $18,09 \pm 2,75 \mathrm{~b}$ \\
Amostra 3 & 5 & 18 & CAv & $46,12 \pm 3,04 \mathrm{~b}$ & $9,12 \pm 0,71 \mathrm{~b}$ & $23,21 \pm 1,10 \mathrm{a}$ \\
Amostra 4 & 5 & 26 & CAv & $45,03 \pm 2,53 \mathrm{~b}$ & $9,99 \pm 0,95 \mathrm{~b}$ & $22,21 \pm 2,10 \mathrm{a}$ \\
Amostra 5 & 10 & 18 & CAc & $39,50 \pm 0,33 \mathrm{c}$ & $9,72 \pm 0,99 \mathrm{~b}$ & $23,12 \pm 2,77 \mathrm{a}$ \\
Amostra 6 & 10 & 26 & CAc & $39,43 \pm 1,32 \mathrm{c}$ & $9,60 \pm 0,21 \mathrm{~b}$ & $24,12 \pm 3,11 \mathrm{a}$ \\
Amostra 7 & 10 & 18 & CAv & $38,03 \pm 0,77 \mathrm{c}$ & $12,27 \pm 2,21 \mathrm{a}$ & $24,39 \pm 3,01 \mathrm{a}$ \\
Amostra 8 & 10 & 26 & CAv & $38,04 \pm 1,45 \mathrm{c}$ & $12,22 \pm 1,31 \mathrm{a}$ & $25,70 \pm 4,21 \mathrm{a}$ \\
\hline
\end{tabular}

a - Teor de glicerol fixado em $20 \mathrm{~g} / 100 \mathrm{~g}$ de sólidos.

${ }^{\mathrm{b}}$ - Letras minúsculas diferentes, na mesma coluna, indicam diferença significativa entre as amostras (Teste de Tukey, $\mathrm{p} \leq 0,05$ ).

${ }^{\mathrm{c}}-\mathrm{CAc}=$ cana-de-açúcar e CAv = casca de aveia.

A coordenada de cromaticidade $\mathrm{a}^{*}$ das amostras apresentou valores que variaram de 1,60 a 12,22 (Tabela 4) e, assim como para a luminosidade, a adição de fibras aumentou significativamente $(\mathrm{p} \leq 0,05)$ esse parâmetro e a umidade não foi significativa. Para essa coordenada, a adição das fibras de casca de aveia, nos maiores teores, levou a um aumento significativo das respostas em comparação à adição das fibras da cana. Considerando que esse componente de cor varia do verde (-60) ao vermelho $(+60)$, pode-se dizer que ocorreu pequena variação para o vermelho entre as amostras, sendo que as amostras com fibras de casca de aveia, nos maiores teores, foram as que apresentaram maior tendência para a cor vermelha. 
A coordenada de cromaticidade $b^{*}$ das amostras apresentou valores que variaram de 7,25 a 25,70 (Tabela 4), sendo que as amostras apresentaram valores significativamente maiores quando adicionadas de fibras ( $\mathrm{p} \leq 0,05)$. A umidade não interferiu nessa resposta e, quanto ao tipo de fibra, a adição das fibras da casca de aveia levou a um aumento da coloração amarelada, quando essas fibras foram empregadas na concentração de 5\%, em comparação às fibras da cana (Tabela 4). Esse componente de cor varia do azul (-60) ao amarelo (+60) e as amostras, de uma forma geral, apresentaram uma tendência em relação à coloração amarelada, em especial quando adicionadas de fibras.

A cor adquirida pelos produtos extrusados está relacionada à caramelização e à reação de Maillard (Menegassi et al., 2007), principalmente em materiais com teores relativamente altos de carboidratos e com proteínas presentes (Gutkoski \& El-Dash, 1999), como é o caso dos materiais empregados neste trabalho. Provavelmente, a adição das fibras tenha levado ao escurecimento das amostras, com decréscimo da luminosidade e aumento dos parâmetros a* e b* em função do aumento do teor de açúcares livres, associados a estas matérias-primas, especialmente no caso das fibras de cana-de-açúcar, que podem conter resíduos de sacarose livre. No caso das fibras de aveia, que nos maiores teores levaram a uma coloração amarelada e avermelhada mais intensa dos compósitos, o que pode ter ocorrido é que esses materiais são originalmente mais escuros que o amido de mandioca e as fibras da cana. De acordo com Salgado et al. (2008), a cor original das matérias-primas influencia diretamente na cor final de espumas de amido de mandioca reforçadas com fibras celulósicas e proteínas.

\section{CONCLUSÕES}

Mediante os resultados observa-se que as fibras podem ser usadas no reforço dos compósitos. A adição das fibras não afetou significativamente o índice de expansão e diminuiu o índice de solubilidade em água, proporcionando, portanto, vantagens para os compósitos. Os compósitos escureceram com a adição de fibras, apresentando menores valores de luminosidade e tendência para coloração avermelhada e amarelada.

Os compósitos biodegradáveis ainda necessitam de condições de processo que forneçam resultados reprodutíveis de expansão e capacidade de absorção e solubilidade em água, propriedades de grande importância nos produtos e para sua produção em escala industrial.

\section{AGRADECIMENTOS}

Ao CNPq (Projeto 478109/2008-3), pelo auxílio financeiro e pela Bolsa de iniciação científica.

\section{REFERÊNCIAS BIBLIOGRÁFICAS}

ALVES, V.D.; MALI, S.; BELÉIA, A.; GROSSMANN, M.V.E. Effects of glycerol and amylose enrichment on cassava starch films propreties. Journal of Food Engineering, Kidlington Oxford, v.78, p.941-946, 2007.

ANDERSON, R.A.; CONWAY, H.F.; PFEIFER, V.F.; GRIFFIN JUNIOR, E.L. Gelatinization of corn grits by roll-and extrusion-cooking. Cereal Science Today, Minneapolis, v.14, n.1, p.44-11, 1969.

BARDI, M.A.G.; ROSA, D.S. Avaliação da biodegradação em solo simulado de poli (ecaprolactona), acetato de celulose e suas blendas.

Revista Brasileira de Aplicações de Vácuo, São Paulo, v.26, n.1, p.43-47, 2007.

CAMARGO, K.F.; LEONEL, M.; MISCHAN, M.M. Produção de biscoitos extrusados de polvilho azedo com fibras: efeito de parâmetros operacionais sobre as propriedades físicas. Ciência e Tecnolologia de Alimentos, Campinas, v.28, n.3, set. 2008.

CARR, L.G.; PARRA, D.F.; PONCE, P.; LUGÃO, A.B.; BUCHLER, P.M. Influence of fibers on the mechanical properties of cassava starch foams. Journal of Polymers and the Environment, Amsterdam, v.14, n.2, p.179-183, 2006.

CINELLI, P.; CHIELLINI, E.; LAWTON, J.W.; IMAN, S.H. Foamed articles based on potato starch, corn fibers and poly(vinyl-alcohol). Polymer Degradation and Stability, Kidlington Oxford, v.91, v.5, p.1147-1155, 2006.

FAMÁ, L.; GERSCHENSON, L.; GOYANES, S. Starchvegetable fibre composites to protect food products. Carbohydrate Polymers, Oxford, v.75, p.230-235, 2009.

FAUBION, J.M.; HOSENEY, R.C. High temperature and short time: extrusion-cooking of wheat starch and flour: I., effect of moisture and flour type on extrudate properties. Cereal Chemistry, Saint Paul, v.59, p.529$533,1982$. 
GLENN, G.M.; ORTS, W.J.; NOBES, A.R. Starch, fiber and $\mathrm{CaCO}_{3}$ effects on the physical properties of foams made by a baking process. Industrial crops and products, Amsterdam, v.14, n.3, p.201-212, 2001.

GUJSKA, E.; KHAN, K. Functional properties of extrudates from high starch fractions of navy and pinto beans and corn meal blended with legume high protein fractions. Journal of Food Science, Chicago, v.56, n.2, p.431-435, 1991

GUTKOSKI, L.C.; EL-DASH, A.A. Effect of extrusion process variables on physical and chemical properties of extruded oat products. Plant foods for human nutrition, Amsterdam, v.54, n.4, p.315-325, 1999.

KARAM, L.B.; GROSSMANN, M.V.E.; SILVA, R.S.F. Misturas de farinha de aveia e amido de milho com alto teor de amilopectina para produção de "snacks". Ciência e Tecnologia de Alimentos, Campinas, v.21, n.2, 2001.

LIMA, S.L.T. Reciclagem e biodegradação de plásticos. Revista Científica do IMAPES, Sorocaba, v.1, p.28-34, 2004.

LUE, S.; HSIEH, F.; HUFF, H.E. Extrusion cooking of corn meal and sugar beet fiber: effects of expansion properties, starch gelatinization and dietary fiber content. Cereal chemistry, Saint Paul, v.63, n.3, p.227234, 1991.

LUSTOSA, B.H.B.; LEONEL, M.; MISCHAN, M.M. Efeito de parâmetros operacionais na produção de biscoitos extrusados de farinha de mandioca. Brazilian Journal of Food Technology, Campinas, v.11, n.1, p.12-19, 2008.

MALI, S.; GROSSMANN, M.V.E.; GARCÍA, M.A.; MARTINO, M.M.; ZARITZKY, N.E. Effects of controlled storage on thermal, mechanical and barrier properties of plasticized fillms from different starch sources. Journal of Food Engineering, Kidlington Oxford, v.75, p.453-460, 2006.

MALI, S.; GROSSMANN, M.V.E.; GARCÍA, M.A.; MARTINO, M.M.; ZARITZKY, N.E. Mechanical and thermal properties of yam starch films. Food

Hydrocolloids, Kidlington Oxford, v.19, p.157-164, 2005.
MALI, S.; GROSSMANN, M.V.E.; YAMASHITA, F. Filmes de amido: produção, propriedades e potencial de utilização. Semina Agrárias, Londrina, v.31, n.1, p.137156,2010 .

MALI, S.; KARAM, L.B.; RAMOS, L.P.; GROSSMANN, M.V.E. Relationships among the composition and physicochemical properties of starches with the characteristics of their films. Journal of Agricultural and Food Chemistry, Easton, v.52, p.7720-7725, 2004.

MENEGASSI, B.; LEONEL, M.; MISCHAN, M. M.; PINHO, S. Z. de. Efeito de parâmetros de extrusão na cor e propriedades de pasta da farinha de mandioquinhasalsa (Arracacia xanthorrhiza). Ciência e Agrotecnologia, Lavras, v. 31, n. 6, p. 1780-1792, Nov./ dez., 2007.

SALGADO, P.R.; SCHMIDT, V.C.; ORTIZ, S.E.M.; MAURI, A.N.; LAURINDO, J. Biodegradable foams based on cassava starch, sunflower proteins and cellulose fibers obtained by a baking process. Journal of Food Engineering, Kidlington Oxford, v.85, p.435-443, 2008.

SCHMIDT, V.C.R.; LAURINDO, J.B. Characterization of foams obtained from cassava starch, cellulose fibres and dolomitic limestone by a thermopressing process. Brazilian Archives of Bioology and Technology, Curitiba, v.53, n.1, p.185192, 2010.

SHIBATA, S.; CAO, Y.; FUKUMOTO, I. Press forming of short natural fiber-reinforced biodegradable resin: effects of fiber volume and length on flexural properties. Polymer Testing, Kidlington Oxford, v.24, n.8, p.10051011, 2005.

SHIMAZU, A.A.; MALI, S.; GROSSMANN, M.V.E. Efeitos plastificante e antiplastificante do glicerol e do sorbitol em filmes biodegradáveis de amido de mandioca. Semina: Ciências Agrárias, Londrina, v.28, p.79-88, 2007.

SHOGREN, R.L.; LAWTON, J.W.; DOANE, W.M.; TIEFENBACHER, K.F. Structure and morphology of baked starch foams. Polymer, Kidlington Oxford, v.39, n.25, p.6649-6655, 1998. 
SILVA, V.L.M.M.; GOMES, W.C.; ALSINA, O.L.S. Utilização do bagaço de cana de açúcar como biomassa adsorvente na adsorção de poluentes orgânicos. Revista Eletrônica de Materiais e Processos, Campina Grande, v.2, p.27-32, 2007.

SILVA, W. A. da; PEREIRA, J.;CARVALHO, C. W. P. de; FERRUA, F.Q. Determinação da cor, imagem superficial topográfica e ângulo de contato de biofilmes de iferentes fontes de amido. Ciência e Agrotecnologia, Lavras, v. 31, n. 1, p.154-163, jan/fev., 2007.

ZHANG, Y.; YANFUL, E.; BASSI, A.S. A review of plastic waste biodegradation. Critical Reviews in

Biotechnology, v.5, n.4, p.243-250, 2005. 